Hum. Genet. 44, 123-135 (1978)

\title{
On the Peripheral Location of the Y Chromosome*
}

\author{
Charles J. Kowalski ${ }^{1 * *}$, C. E. Nasjleti ${ }^{2}$, and Becky Schmitt ${ }^{2}$ \\ ${ }^{1}$ Dental Research Institute, 1034, Kellogg Building, University of Michigan, Ann Arbor, \\ MI 48109, USA \\ ${ }^{2}$ Veterans Administration Hospital, Ann Arbor, Michigan, USA
}

Summary. The location of the $\mathrm{Y}$ chromosome in metaphase spreads is studied in groups of (a) 96 normal males, (b) 17 Down's syndrome patients, and (c) 51 patients with Klinefelter's syndrome. The position of the $Y$ is scored as either peripheral or nonperipheral using several of the traditional definitions found in the literature. It is shown that contradictory results may be obtained depending on the particular definition employed and that none of the available definitions capture the essence of the meaning of 'peripheral.' Therefore a new, standardized definition is introduced-one that applies to both circular and noncircular spreads. Using this new definition, we find definite intergroup variability. The $\mathrm{Y}$ is peripheral in normal males, but not in either the Down's or Klinefelter's samples.

\section{Introduction}

There is now much evidence that chromatin is attached to the nuclear membrane, that at least some of the attachment sites correspond to points of initiation of DNA replication, and that there is some order to the arrangement of chromatin in the interphase nucleus (Comings, 1968). Thus, although the air-drying and squash techniques of spreading metaphase chromosomes are disruptive procedures, if the interphase chromatin is arranged according to some definite pattern, the relative positions of the chromosomes in metaphase preparations should tend to be nonrandom. While this is generally accepted, there is less agreement about the actual nonrandom positions assumed by human metaphase chromosomes. A case in point is the $\mathrm{Y}$ chromosome. While the consensus apparently favors the notion that the $\mathrm{Y}$ chromosome is located peripherally more often than expected by chance alone (Barton et al., 1965; Miller et al., 1963a and b), there have been some dissenters (Gripenberg, 1964; Spence et al., 1973) and even some who have been able simultaneously to agree and disagree with the underlying hypothesis (Cohen et al., 1966; Ockey, 1969).

* Supported, in part, by the Medical Research Service of the Veterans Administration

** To whom offprint requests should be sent 


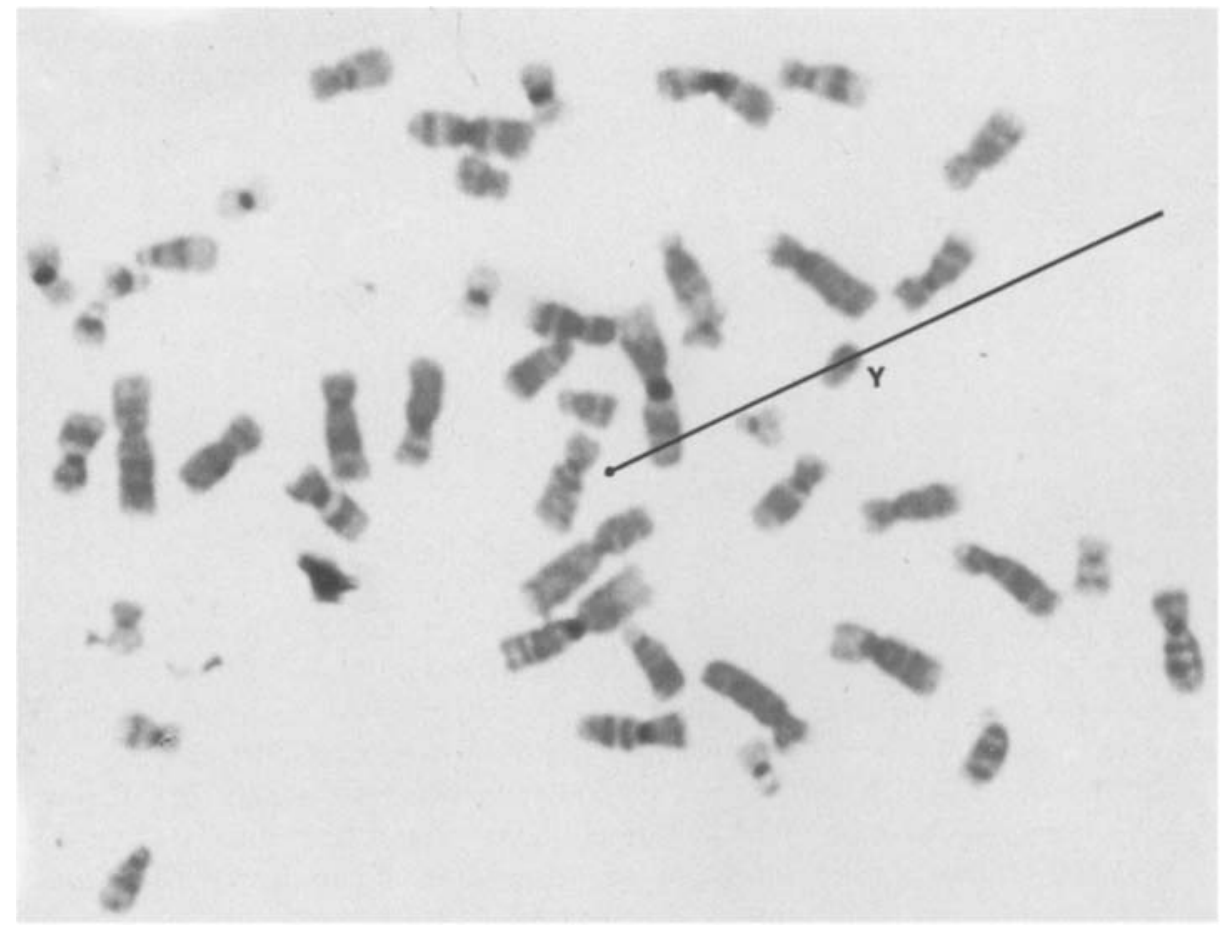

Fig. 1. The $Y$ chromosome is peripheral according to Gripenberg's definition, although it lies near the center of the metaphase plate

Problems inhibiting the reconciliation of the conflicting claims include lack of a standard definition of peripheral and lack of standardization of sample sizes, techniques, and populations studied. More generally, potential bias may be introduced by selecting only circular spreads for study and by obtaining metaphases from only a few individuals. Thus, although Spence et al. (1973) recognized the possibility that "there are differences between individuals in chromosome association patterns" (Back and Zang, 1969; Cooke, 1971), their evidence against the peripheral location of the $\mathrm{Y}$ chromosome (and, indeed, against the existence of interindividual differences) was based on but two normal males and one XYY individual.

Similar confusion exists concerning the location of the autosomes. For example, Barton et al. (1965) found that chromosomes 13-15, 21, and 22 lie near the center of metaphase spreads, contradicting the earlier work of Miller et al. (1963b).

The purpose of this paper is to present new data bearing on these questions. First, we reexamined the location of the Y chromosome by employing Gripenberg's (1964) definition of peripheral in (a) male controls, (b) Down's syndrome patients $(47, \mathrm{XY},+21)$, and (c) persons with Klinefelter's syndrome $(47, \mathrm{XXY})$. Besides the $Y$, each of the other metaphase chromosomes, identified by trypsin banding, are similarly classified as peripheral or nonperipheral in each group. 
Second, we devised a new, standardized, and easily reproduced definition of peripheral to study the location of the $\mathrm{Y}$ chromosome in each of the three groups defined above.

The need for a new definition of peripheral should be apparent from a glance at Figure 1: the Y chromosome's position is surely such that most people would not describe it as peripheral; yet according to Gripenberg's definition, it is. The reverse situation also frequently occurs, i.e., the $Y$ is often near the periphery of the spread; yet it is not counted as peripheral simply because a single chromosome is located further out on the radius. Nor are other previously reported definitions of peripheral entirely satisfactory. Most are applicable only to circular spreads and require that the center of the circle be determined by eye. Others involve circular definitions in the sense that the decision as to whether or not the $\mathrm{Y}$ is peripheral depends on its position relative to other peripherally located chromosomes - but how these other chromosomes were classified as peripheral is not indicated.

\section{Materials and Methods}

Cells were cultured from peripheral blood of individuals from three groups: (a) 96 normal adult males, 20-40 years old, most employed at the Veterans Administration Hospital in Ann Arbor, Michigan; their karyotypes were previously used by Harris et al. (1973) and by Kowalski et al. (1976) for other purposes; (b) 17 Down's syndrome patients, 2-14years old; and (c) 51 patients with Klinefelter's syndrome. The latter two groups were obtained from the University of Michigan Medical Center.

The cultures were processed essentially following the technique of Moorhead et al. (1960), the final spreading of the chromosomes being achieved by drying in air. The slight modifications that we introduced to this method have been described elsewhere (Nasjleti et al., 1966). The chromosomes were stained with Giemsa and the banding patterns were produced by the trypsin method of Seabright (1971).

Each of the chromosomes in the metaphase spreads was then classified as either peripheral or nonperipheral according to Gripenberg's criteria (1964); viz, a chromosome is peripheral if there is no other chromosome further from the center on the same radius than the centromere of the chromosome in question. This is illustrated in Figure 2, where a typical metaphase spread is shown and the numbered chromosomes counted as being peripheral according to the above definition are indicated. The radius shown passes through the centromere of the Ychromosome but does not intersect a chromosome further from the center, establishing its peripheral location in this instance.

Both the chi-square test and Fisher's exact test for association in $2 \times 2$ contingency tables were employed to test the hypothesis that the Y chromosome is peripherally located more often than the other chromosomes in the metaphase spreads. While most have tested this hypothesis with chi-square alone, Fisher's exact test is preferred in the case of $2 \times 2$ tables since the exact distribution of the test statistic is known, whereas the chi-square distribution is but an approximation (Kowalski, 1970). These tests are done within each of the three groups defined previously.

An alternative definition of peripheral was devised and employed to reexamine the $Y$ chromosome in metaphase spreads. For this, we define the horizontal plane for the metaphase spread by drawing a line connecting the centromeres of the chromosomes 1 . The choice of this particular pair of chromosomes is entirely arbitrary; any other pair would serve equally well, but the chromosomes 1 are easy to identify and generally have well-defined centromeres. Lines parallel to this baseline are then drawn through the centromeres of the chromosomes most peripheral with respect to this starting point (Fig. 3). These lines have the property that no 

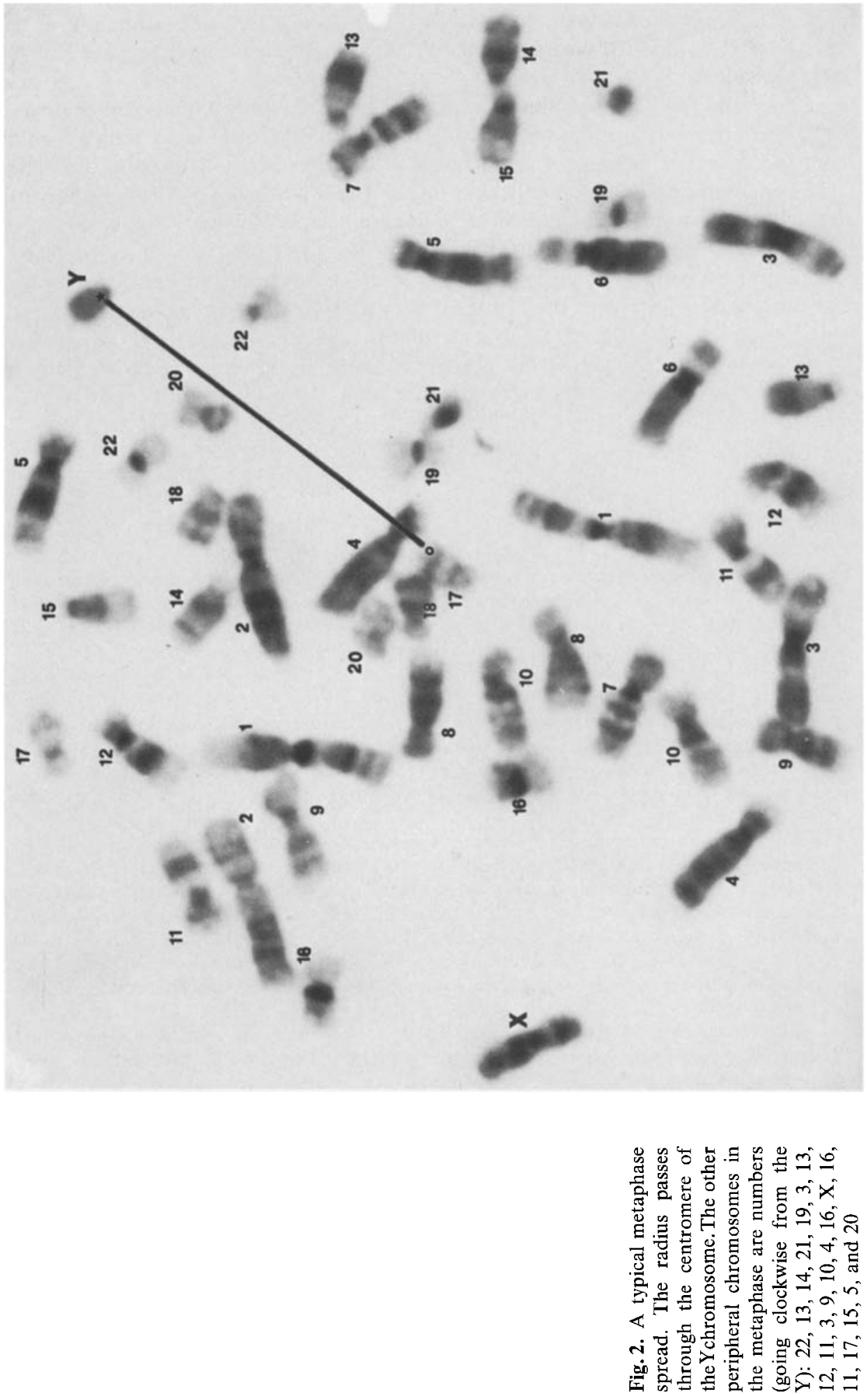


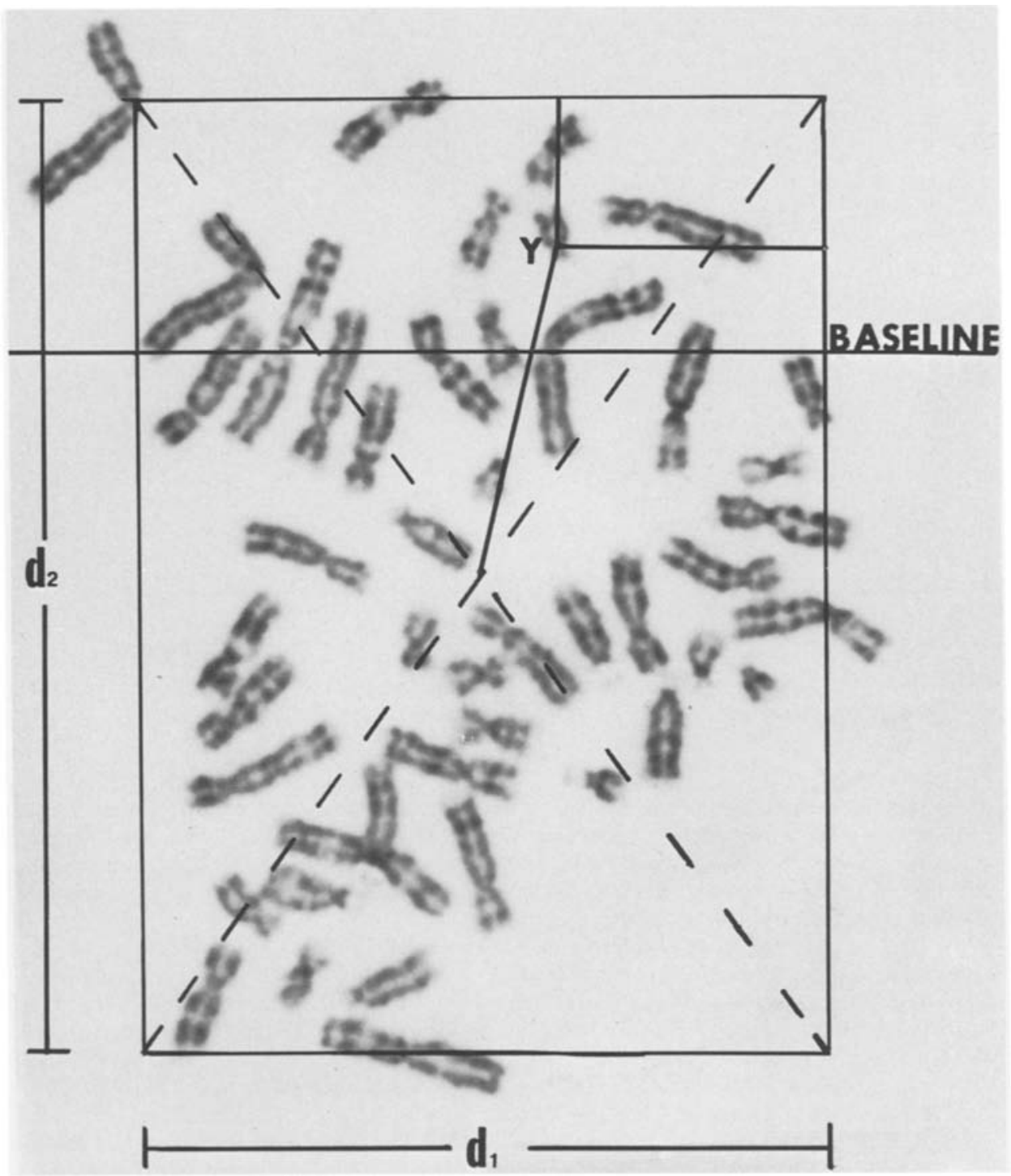

Fig. 3. How the bounding rectangle used in our definition of peripheral is constructed

chromosome has a centromere lying beyond them in the vertical direction. A rectangle is then formed by drawing lines perpendicular to the others, such that the centromeres of all of the chromosomes in the spread are in (or on) the rectangle. This procedure can be applied to metaphase spreads of any kind-not just circular spreads - and is entirely reproducible in other laboratories. Providing only that we agree on the same starting point (the homologous pair of chromosomes 1), the rectangle is completely specified. The center of the rectangle is well defined and easy to locate: simply draw the diagonals in the rectangle and mark their point of intersection. When this is done on a circular spread, the derived center agrees closely with the optical center, and the rectangle becomes a square within which the circle is inscribed. Thus, for circular spreads, results should closely correspond to those obtained using procedures based on other 


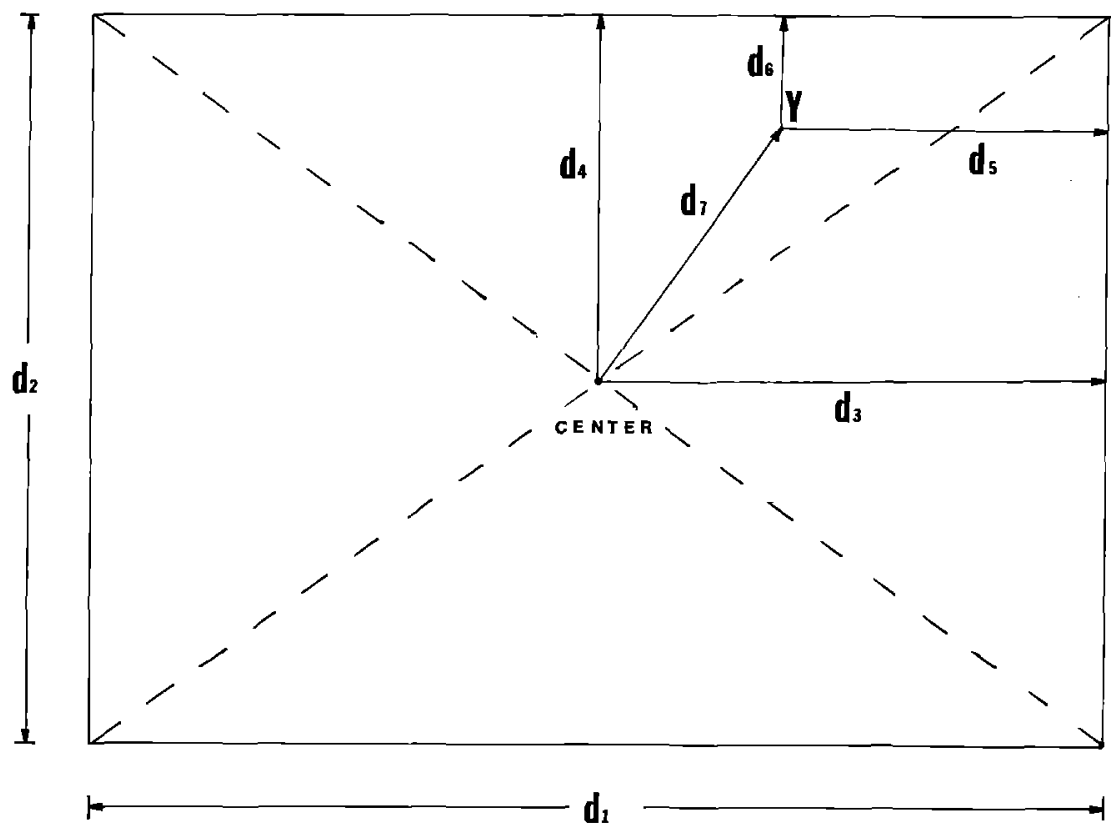

Fig. 4. Some measurements that may help characterize the position of the $\mathrm{Y}$ chromosome relative to the bounding rectangle

definitions of peripheral that are limited to such circular metaphase plates. However, the method also easily accomodates noncircular spreads. This may be seen as a distinct advantage inasmuch as our intuitive appreciation of peripheral does not require an underlying circular structure. A chromosome near the boundary of the rectangle should be counted as peripheral whether or not the spread is circular.

It remains to specify what we mean by near the boundary of the rectangle. This is, of course, again arbitrary and depends on the number of zones we wish to distinguish between within the derived rectangle. Basically, zones may be defined by rectangles having the same center as the bounding rectangle and proportionate sides. If, e.g., we wanted just two zones with equal areas, we could inscribe a rectangle with sides $1 / \sqrt{2}$ times the sides of the bounding rectangle. Using the notation defined in Figure 4 , the inner rectangle would then have area $d_{1} / \sqrt{2} \cdot d_{2} / \sqrt{2}=$ $d_{1} d_{2} / 2$. The outer zone would then have an area equal to the area of the bounding rectangle minus that of the inner zone, viz., $d_{1} d_{2}-d_{1} d_{2} / 2=d_{1} d_{2} / 2$. Of course, more than two zones could be used and not all of the zones need to be equal in area. Nonetheless, once the zones are defined, comparable results can be obtained by anyone using the system. The set of measurements illustrated in Figure 4 may be useful in comparing the location of the $\mathrm{Y}$ chromosome in several groups of individuals: $d_{1}$ and $d_{2}$ reflect the size of the bounding rectangle; $d_{5}$ and $d_{6}$ are the minimal distances of the centromere of the Y chromosome to the bounding rectangle in the horizontal and vertical directions, respectively; $d_{7}$ is the distance from the center of the rectangle to the centromere of the Ychromosome; $d_{3}$ and $d_{4}$ are not measured directly, but are obtained by $d_{3}=d_{1} / 2$ and $d_{4}=d_{2} / 2$. These measurements are useful when comparing $d_{5}$ and $d_{6} ; d_{5} / d_{3}$ and $d_{6} / d_{4}$ take values on the interval $[0,1]$ and express the distance that the Ychromosome lies from the bounding rectangle in the horizontal and vertical directions in standardized units. In a similar manner, we compute $d_{8}$ (not shown on the figure) as the distance from the center to any one of the corners of the rectangle so that $d_{7} / d_{8}$ is standardized on the interval $[0,1]$. Small values indicate that the $\mathrm{Y}$ lies near the center of the spread; large values, near the periphery. Another useful ratio is $d_{5} \cdot d_{6} / d_{3} \cdot d_{4}$. This also takes values on the interval $[0,1]$ and has a direct interpretation in terms of the location of the $\mathrm{Y}$ chromosome. 


\section{Results}

We begin with results obtained using Gripenberg's (1964) definition of peripheral. Table 1 summarizes the findings for each of the chromosomes in each of the groups considered. In each instance, the number of peripheral chromosomes $(\mathrm{P})$, the total number of chromosomes examined (T), and the percentage of peripheral chromosomes $(\% \mathrm{P})$ are given.

It is seen that the Y chromosome in normal males is peripheral in $62.5 \%$ of the cells studied. Table 2 shows the results of the test of the hypothesis that the $Y$ chromosome is peripherally located more often than the other chromosomes in the metaphase spreads. The $P$ values corresponding to both the chi-square test and Fisher's exact test are given. Both $P$ values are zero correct to four decimal places, clearly establishing the peripheral location of the $\mathrm{Y}$ chromosome in normal males.

Table 1. Numbers of peripheral chromosomes $(\mathrm{P})$; total number of particular chromosome studied $(\mathrm{T})$; and the percentage of peripheral chromosomes $(\% \mathrm{P})$ : in controls, Down's syndrome, and Klinefelter's syndrome

\begin{tabular}{|c|c|c|c|c|c|c|c|c|c|}
\hline \multirow{2}{*}{$\begin{array}{l}\text { Chromo- } \\
\text { some }\end{array}$} & \multicolumn{3}{|c|}{ Controls } & \multicolumn{3}{|c|}{ Down's syndrome } & \multicolumn{3}{|c|}{ Klinefelter's syndrome } \\
\hline & $\mathrm{P}$ & $\mathrm{T}$ & $\% \mathrm{P}$ & $\mathrm{P}$ & $\mathrm{T}$ & $\% \mathrm{P}$ & $\mathrm{P}$ & $\mathrm{T}$ & $\% \mathrm{P}$ \\
\hline 1 & 48 & 192 & 25.0 & 10 & 34 & 29.4 & 32 & 102 & 31.2 \\
\hline 2 & 46 & 192 & 23.4 & 12 & 34 & 35.3 & 30 & 102 & 29.4 \\
\hline 3 & 67 & 192 & 34.9 & 12 & 34 & 35.3 & 35 & 102 & 34.3 \\
\hline 4 & 56 & 192 & 29.2 & 12 & 34 & 35.3 & 33 & 102 & 32.4 \\
\hline 5 & 63 & 192 & 32.8 & 7 & 34 & 20.6 & 33 & 102 & 32.4 \\
\hline 6 & 61 & 192 & 31.8 & 13 & 34 & 38.2 & 32 & 102 & 31.2 \\
\hline 7 & 81 & 192 & 42.2 & 11 & 34 & 32.4 & 33 & 102 & 32.4 \\
\hline 8 & 87 & 192 & 45.3 & 13 & 34 & 38.2 & 26 & 102 & 25.5 \\
\hline 9 & 71 & 192 & 37.0 & 17 & 34 & 50.0 & 31 & 102 & 30.4 \\
\hline 10 & 87 & 192 & 45.3 & 13 & 34 & 38.2 & 36 & 102 & 35.3 \\
\hline 11 & 86 & 192 & 44.8 & 9 & 34 & 26.5 & 19 & 102 & 18.6 \\
\hline 12 & 74 & 192 & 38.5 & 7 & 34 & 20.6 & 34 & 102 & 33.3 \\
\hline 13 & 79 & 192 & 41.1 & 7 & 34 & 20.6 & 25 & 102 & 24.5 \\
\hline 14 & 74 & 192 & 38.5 & 10 & 34 & 29.4 & 31 & 102 & 30.4 \\
\hline 15 & 71 & 192 & 37.0 & 14 & 34 & 41.2 & 29 & 102 & 28.4 \\
\hline 16 & 68 & 192 & 35.4 & 14 & 34 & 41.2 & 23 & 102 & 22.5 \\
\hline 17 & 66 & 192 & 34.4 & 16 & 34 & 47.1 & 19 & 102 & 18.6 \\
\hline 18 & 84 & 192 & 43.8 & 14 & 34 & 41.2 & 31 & 102 & 30.4 \\
\hline 19 & 71 & 192 & 37.0 & 15 & 34 & 44.1 & 23 & 102 & 22.5 \\
\hline 20 & 71 & 192 & 37.0 & 20 & 34 & 58.8 & 25 & 102 & 24.5 \\
\hline 21 & 78 & 192 & 40.6 & 18 & 51 & 35.3 & 24 & 102 & 23.5 \\
\hline 22 & 62 & 192 & 32.3 & 18 & 34 & 52.9 & 29 & 102 & 28.4 \\
\hline $\mathrm{X}$ & 42 & 96 & 43.8 & 3 & 17 & 17.6 & 31 & 102 & 30.4 \\
\hline $\mathrm{Y}$ & 60 & 96 & 62.5 & 3 & 17 & 17.6 & 17 & 51 & 33.3 \\
\hline
\end{tabular}


Table 2. Tests of the hypothesis that the $\mathrm{Y}$ chromosome is peripherally located in normal males

\begin{tabular}{lccr}
\hline & Peripheral & $\begin{array}{l}\text { Non- } \\
\text { peripheral }\end{array}$ & Total \\
\hline Y chromosome & 60 & 36 & 96 \\
Other chromosomes & 1593 & 2772 & 4365 \\
\hline Total & 1653 & 2808 & 4461 \\
\hline
\end{tabular}

$\chi^{2}=26.132$

$P=0.0000$

Fisher's exact test $P=0.0000$

Table 3. Tests of the hypothesis that the $\mathrm{Y}$ chromosome is peripherally located in patients with Down's syndrome

\begin{tabular}{lccc}
\hline & Peripheral & $\begin{array}{l}\text { Non- } \\
\text { peripheral }\end{array}$ & Total \\
\hline Y chromosome & 3 & 14 & 17 \\
Other chromosomes & 285 & 497 & 782 \\
\hline Total & 288 & 511 & 799 \\
\hline
\end{tabular}

$\chi^{2}=1.80$

$P=0.1797$

Fisher's exact test $P=0.0856$

Tables 3 and 4 give the results of the Down's and Klinefelter's syndrome groups, respectively. In both groups, the $Y$ chromosome does not differ significantly from the other chromosomes with respect to the frequency of peripheral location. In the Down's syndrome group, only 17.6\% of the Y chromosomes were found to be peripheral, and the test in this case is really to assess the significance of the nonperipheral location of the Y chromosome. Indeed, our results indicate that the Y may be nonperipheral in patients with Down's syndrome $(P=0.09$ using Fisher's exact test), but the sample size is too small to establish significance.

Also, Table 1 shows that the autosomes in all three groups show no particular pattern with respect to peripheral location. Nor do the extra chromosomes in the nonnormal groups tend to be peripherally located. Chromosome 21 in Down's syndrome was found to be peripheral only $35.3 \%$ of the time, and the X chromosome in the XXY individuals was peripheral in $30.4 \%$ of the cells examined. Peripheral location is not related to the size and/or shapes of the metaphase chromosomes. Only the $\mathrm{Y}$ chromosome in normal males is unquestionably peripheral, most of the other chromosomes being found peripheral about $30-40 \%$ of the time.

The first results obtained using the definition of peripheral developed in this paper are those obtained when the rectangle is divided into two zones, say inner 
Table 4. Tests of the hypothesis that the $\mathrm{Y}$ chromosome is peripherally located in patients with Klinefelter's syndrome

\begin{tabular}{lccc}
\hline & Peripheral & $\begin{array}{l}\text { Non- } \\
\text { peripheral }\end{array}$ & Total \\
\hline Y chromosome & 17 & 34 & 51 \\
Other chromosomes & 663 & 1683 & 2346 \\
\hline Total & 680 & 1717 & 2397 \\
\hline
\end{tabular}

$\chi^{2}=0.407$

$P=0.5235$

Fisher's exact test $P=0.2577$

Table 5. Test of the hypothesis that the percentage of peripheral $\mathrm{Y}$ chromosomes is the same in the three groups, using two zones with equal areas. Numbers in parentheses refer to (row) percentages

\begin{tabular}{lccc}
\hline & Inner zone & Outer zone & Total \\
\hline Controls & 37 & 59 & 96 \\
& $(38.5 \%)$ & $(61.5 \%)$ & \\
Down's syndrome & 13 & 4 & 17 \\
& $(76.5 \%)$ & $(23.5 \%)$ & \\
Klinefelter's syndrome & 30 & 21 & 51 \\
& $(58.8 \%)$ & $(41.2 \%)$ & \\
\hline
\end{tabular}

$\chi^{2}=11.30$

$P=0.004$

and outer, having equal areas. The chi-square test was used to determine whether the percentage of times the Y chromosome is peripheral is the same in the three groups; the results are summarized in Table 5.

Clear differences are evident between the groups $(P=0.004) ; 61.5 \%$ of the Y chromosomes in normal males were peripheral, $23.5 \%$ in the Down's syndrome sample, and $41.2 \%$ among those with Klinefelter's syndrome. Other definitions of outer, inner, and intermediate zones produced analogous results. While the percentages changed, the differences between the groups were maintained.

Some of the other measurements designed to assess the location of the $\mathrm{Y}$ chromosome (Fig. 4) can be analyzed by the analysis of variance. The results for the ratio $d_{5} \cdot d_{6} / d_{3} \cdot d_{4}$ are shown in Table 6 . The analysis of variance (significant, with $P=0.009$ ) was followed by Scheffé's multiple comparison procedure, which compares the three groups by pairs. This showed that the normal males differed significantly from the Down's group, but the differences between other pairs were not significant at the $5 \%$ level.

The same test was used to compare the mean values of the ratio $d_{7} / d_{8}$ in the three groups. Again, the hypothesis that the means are the same in each of the 
Table 6. Means and variances of the ratio $d_{5} \cdot d_{6} / d_{3} \cdot d_{4}$ in the three groups and the $P$ value for the (analysis of variance) test that the means equal

\begin{tabular}{llll}
\hline Group & $n$ & Mean & Variance \\
\hline Controls & 96 & 0.22 & 0.052 \\
Down's syndrome & 17 & 0.40 & 0.084 \\
Klinefelter's syndrome & 51 & 0.29 & 0.055 \\
\hline
\end{tabular}

$P=0.009$

Table 7. Means and variances of the ratio $d_{7} / d_{8}$ in the three groups and the $P$ value for the (analysis of variance) test that the means equal

\begin{tabular}{lccl}
\hline Group & $n$ & Mean & Variance \\
\hline Controls & 96 & 0.56 & 0.040 \\
Down's syndrome & 17 & 0.40 & 0.044 \\
Klinefelter's syndrome & 51 & 0.48 & 0.037 \\
\hline
\end{tabular}

$P=0.004$

Table 8. Chi-square test based on zones defined by the values of the ratio $d_{7} / d_{8}$. Numbers in parentheses refer to (row) percentages

\begin{tabular}{lcccc}
\hline \multirow{2}{*}{ Group } & \multicolumn{2}{c}{ Zone } & & \multirow{2}{*}{ Total } \\
\cline { 2 - 4 } & Inner & Middle & Outer & \\
\hline Controls & 17 & 48 & 31 & 96 \\
& $(17.7 \%)$ & $(50.0 \%)$ & $(32.3 \%)$ & \\
Down's syndrome & 7 & 9 & 1 & 17 \\
Klinefelter's syndrome & $(41.2 \%)$ & $(52.9 \%)$ & $(5.9 \%)$ & \\
& 11 & 30 & 10 & 51 \\
\hline
\end{tabular}

$\chi^{2}=9.19$

$P=0.056$

three groups was rejected $(P=0.004)$, and again, only the normal males and Down's group differed significantly at the $5 \%$ level. The results are summarized in Table 7.

We also did a chi-square test on this variable by dividing its range from zero to one into third thirds and counting the number of times this ratio took values in each subinterval. The results are shown in Table 8 , and again, the most striking difference is noted between the normal males and the Down's group. The columns in the table are labeled inner, middle, and outer according to whether the ratio was in the interval $[0,1 / 3],[1 / 3,2 / 3]$, or $[2 / 3,1]$, respectively. 
Table 9. Chi-square test based on zones defined by the values of the values of the minimum of the ratios $d_{5} / d_{3}$ and $d_{6} / d_{4}$. Numbers in parentheses refer to (row) percentages

\begin{tabular}{lcccc}
\hline \multirow{2}{*}{ Group } & \multicolumn{2}{c}{ Zone } & \multirow{2}{*}{ Total } \\
\cline { 2 - 4 } & Inner & Middle & Outer & \\
\hline Controls & 12 & 23 & 61 & \multirow{2}{*}{96} \\
& $(12.5 \%)$ & $(24.0 \%)$ & $(63.5 \%)$ & \\
Down's syndrome & 6 & 5 & 6 & 17 \\
Klinefelter's syndrome & $(35.3 \%)$ & $(29.4 \%)$ & $(35.3 \%)$ & \\
& 7 & 23 & 21 & 51 \\
\hline
\end{tabular}

$\chi^{2}=14.14$

$P=0.007$

This analysis was repeated for the variable defined as the minimum of the ratios $d_{5} / d_{3}$ and $d_{6} / d_{4}$ (Table 9). Here, small values for the resulting variable indicate peripheral location (outer zone) of the $\mathrm{Y}$ chromosome, but the results otherwise correspond to those shown in Table 8.

A number of additional analyses, each based on some transformation of the variables defined in Figure 5, were done, and each showed essentially the same pattern: the Y chromosome is clearly peripheral in control metaphases, and the proportion of peripheral Y's in the group differs significantly from that found in individuals with Down's syndrome.

\section{Discussion}

Table 1 shows that the $\mathrm{Y}$ chromosome in normal males was peripheral, according to Gripenberg's definition, in $62.5 \%$ of the cells studied. This is more often than would be expected by chance alone ( Table 2) and is much more often than has been reported by most other investigators. While Miller et al. (1963a) found the $Y$ to be peripheral in $62 \%$ of the metaphases obtained from X0/XY mosaics and in $56.8 \%$ of their pooled sample, most other studies have produced less decisive results. Cohen et al. (1966), for example, found $44 \%$ when their questionably peripheral chromosomes were counted as peripheral, but only $32.7 \%$ when not. While the former figure is not significantly different from the results of Miller et al. (1963a), the latter is. Thus, at least part of the confusion here may be explained by the different difinitions of peripheral in the two studies. Gripenberg (1964) reported the $\mathrm{Y}$ to be peripherally located only $33.3 \%$ of the time, and Ockey (1969) found the $\mathrm{Y}$ in his outer zone about $30 \%$ of the time. Although this figure was somewhat less than the random expected value (34.8\%), Ockey (1969) concluded that the $\mathrm{Y}$ was "significantly more peripheral than the early synthesizing chromosomes of similar length." Gripenberg (1964) might have argued more strongly against the peripheral location of the Y. Given here data, the correct value of the chi-square is 0.215 , corresponding to $P=0.64$, which is much larger 
than the $P<0.30$ she reported. In any event, having employed the same definition of peripheral as Gripenberg (1964), our results disagree with hers and tend to support the findings of Miller et al. (1963a and b) and Barton et al. (1965) concerning the peripheral location of the $\mathrm{Y}$ chromosome in control cells.

On the other hand, Miller et al. (1963a) mentioned an unpublished study by Breg and Miller, which found the Y chromosome peripherally located in trisomy 21 patients. Our findings do not support this contention. Nor can we support the evidence advanced by Miller et al. (1963b) that chromosomes 13, 17, 18, and 21 are peripherally located. Indeed, Table 1 incicates that none of the autosomes tends to be located peripherally. While chromosomes 13 and 18 were peripheral in $40.7 \%$ and $43.3 \%$ of the normal control metaphases, respectively, these percentages are no more than those observed for chromosomes $7,8,10$, and 11 .

These observations, of course, depend on our definition of peripheral and our results are directly comparable only with those reported by Gripenberg (1964). Thus, we feel that it is important to standardize the definition of peripheral and have offered one such possibility in this paper.

Perhaps the easiest definition in this context is the one based on just two zones having equal areas. In this case (Table 5), $61.5 \%$ of the $\mathrm{Y}$ chromosome in controls were peripheral and this finding can be compared with the results obtained by other laboratories (or in samples from other populations of individuals) provided only that this simple definition is implemented. While other definitions of peripheral are possible (Tables 6-9) using the measurements defined in Figure 5, the simple definition should suffice for most applications. In any event, the differences noted between the three groups under consideration are consistent irrespective of which definition of peripheral is employed. When the analysis of variance was used (Tables 6 and 7), multiple comparison procedures showed that the Klinefelter's group did not differ significantly from either of the other two groups. However, such procedures are known to have but limited sensitivity and are all but nonexistent for "finding where the significance lies" in the chi-square test context (Kowalski, 1971). Thus we prefer not to attempt to characterize the differences between the three groups in any final way.

\section{References}

Back, E., Zang, K. D.: Quantitative studies on the arrangement of human metaphase chromosomes. II. Influence of the preparation technique on the association pattern of the acrocentric chromosomes. Cytogenetics 8, 304-314 (1969)

Barton, D. E., David, F. N., Merrington, M.: The relative position of the chromosomes in the human cell in mitosis. Ann. Hum. Genet. 29, 139-146 (1965)

Cohen, M. M., Shaw, M. W., MacCluer, J. W.: Racial differences in the length of the human Y chromosome. Cytogenetics 5, 34-52 (1966)

Comings, D. E.: The rationale for an ordered arrangement of chromatin in the interphase nucleus. Am. J. Hum. Genet. 20, 440-460 (1968)

Cooke, P.: Non-random participation of chromosomes 13, 14, and 15 in acrocentric associations. Humangenetik 13, 309-314 (1971)

Gripenberg, U.: Size variation and orientation of the human Y chromosome. Chromosoma $15,618-629(1964)$ 
Harris, J. E., Nasjleti, C. E., Kowalski, C. J.: Discrimination between groups of chromosomes and individual chromosomes in the normal karyotype. Chromosoma (Berl.) 40, 269-284 (1973)

Kowalski, C. J.: The performance of some rough tests for bivariate normality before and after coordinate transformations to normality. Technometrics 12, 517-544 (1970)

Kowalski, C. J.: Relationship between smoking and calculus deposition. J. Dent. Res. 50, 101-104 (1971)

Kowalski, C. J., Nasjleti, C. E., Harris, J. E.: Human chromosomes. Evidence for autosomal sexual dimorphism. Exp. Cell Res. 100, 56-62 (1976)

Miller, O. J., Mukherjee, B. B., Breg, W. R., van Gamble, A.: Non-random distribution of chromosomes in metaphase figures from cultured human leukocytes. I. The peripheral location of the Y chromosome. Cytogenetics 2, 1-14 (1963a)

Miller, O. J., Breg, W. R., Mukherjee, B. D., van Gamble, A., Christakos, A. C.: Non-random distribution of chromosomes in metaphase figures from cultured human leukocytes. II. The peripheral location of chromosomes 13, 17-18 and 21. Cytogenetics 2, 152-168 (1963b)

Moorhead, P. S., Nowell, P. C., Mellman, W. J., Battips, D. D., Hungerford, D. M.: Chromosome preparation of leukocytes cultured from human peripheral blood. Exp. Cell Res. 20, $613-616(1960)$

Nasjleti, C. E., Walden, J. M., Spencer, H. H.: Polyploidization and aberration of human chromosomes induced in vitro and in vivo with ionizing radiations. J. Nucl. Med. 7 , $159-176(1966)$

Ockey, C. H.: The positions of chromosomes at metaphase in human fibroblasts and their DNA synthesis behavior. Chromosoma (Berl.) 27, 308-320 (1969)

Seabright, M.: A rapid banding technique for human chromosomes. Lancet 1971 II, 971—972

Spence, M. A., Francke, U., Forsythe, A. B.: Evidence against the peripheral location of the Y chromosome in human metaphase cells. Cytogenet. Cell Genet. 12, 49-52 (1973)

Received December 29, 1977 